\title{
Restoration of Cdk5, TrkB and Soluble N-ethylmaleimide-Sensitive Factor Attachment Protein Receptor Proteins after Chronic Methylphenidate Treatment in Spontaneous Hypertensive Rats, a Model for Attention-Deficit Hyperactivity Disorder
}

\author{
Yeni Kim ${ }^{1 *} \bowtie$, Songhee Jeon ${ }^{2 *}$, Ha Jin Jeong ${ }^{2}$, Seong Mi Lee ${ }^{1}$, Ike dela Peña ${ }^{3,4}$, \\ Hee Jin $\mathrm{Kim}^{3}$, Doug Hyun Han ${ }^{5}$, Bung-Nyun $\mathrm{Kim}^{6}$, and Jae Hoon Cheong ${ }^{3}$ \\ ${ }^{1}$ Department of Child and Adolescent Psychiatry, National Center for Mental Health, Seoul, Republic of Korea \\ ${ }^{2}$ Department of Biomedical Sciences, Center for Creative Biomedical Scientists at Chonnam National University, Gwangju, Republic of Korea \\ ${ }^{3}$ Department of Pharmacy, Sahmyook University, Uimyung Research Institute for Neuroscience, Seoul, Republic of Korea \\ ${ }^{4}$ Department of Pharmaceutical and Administrative Sciences, Loma Linda University Health, Loma Linda, CA, USA \\ ${ }^{5}$ Department of Psychiatry, Chung Ang University Hospital, Seoul, Republic of Korea \\ ${ }^{6}$ Division of Child \& Adolescent Psychiatry, Department of Psychiatry and Institute of Human Behavioral Medicine, Seoul National University \\ College of Medicine, Seoul, Republic of Korea
}

Objective Synaptic vesicle mobilization and neurite outgrowth regulation molecules were examined in modulation of effects of methylphenidate (MPH) in Spontaneous Hypertensive Rats (SHRs), a model for attention-deficit hyperactivity disorder (ADHD).

Methods We compared the changes in the protein expression level of Cyclin dependent kinase 5 (Cdk5) and molecular substrates of Cdk5; tropomyosin receptor kinase B (TrkB), syntaxin 1A (STX1A) and synaptosomal-associated protein 25 (SNAP25). Comparisons were made in prefrontal cortex of vehicle (distilled water i.p. for 7 days)-treated SHRs, vehicle-treated Wistar Kyoto Rats (WKYs) and MPH (2 mg/kg i.p. for 7 days) treated SHRs.

Results The Cdk5 level of vehicle-treated SHRs was significantly decreased compared to the Cdk5 level of vehicle-treated WKY rats, but was restored to the expression level of vehicle-treated WKYs in MPH-treated SHR. The ratio of p25/p35 was significantly decreased in MPH-treated SHR compared to vehicle-treated SHR. Moreover, TrkB, STX1A and SNAP25 of vehicle-treated SHRs were significantly decreased compared to vehicle-treated WKY rats, but were restored to the expression level of vehicle-treated WKYs in MPH-treated SHR. Conclusion The results show that Cdk5, TrkB, STX1A, and SNAP25 were involved in the modulation of MPH effects in prefrontal cortex of SHRs and play important role in treatment of ADHD. Psychiatry Investig 2019;16(7):558-564

Key Words Methylphenidate, Cyclin dependent kinase 5, Tropomyosin receptor kinase B, Syntaxin 1A, Synaptosomal-associated protein 25 .

\section{INTRODUCTION}

Attention-deficit hyperactivity disorder (ADHD) is a condition that has a worldwide prevalence ranging from $3 \%$ to $8 \%$ in the paediatric population. ${ }^{1,2}$ The core features are inat- tention, hyperactivity and impulsivity. Spontaneously hypertensive rats (SHR) are a genetic model for ADHD bred from progenitor Wistar-Kyoto rats (WKY). ${ }^{3}$ SHR are the most widely utilized animal model for ADHD, especially the combined type ${ }^{4-6}$ since they display sustained attention deficits and hy-

Received: November 29, 2017 Revised: February 23, 2018 Accepted: April 22, 2019

$\bowtie$ Correspondence: Jae Hoon Cheong, $\mathrm{PhD}$

Department of Pharmacy, Sahmyook University, Uimyung Research Institute for Neuroscience, 815 Hwarang-ro, Nowon-gu, Seoul 01795, Republic of Korea Tel: +82-2-3399-1605, Fax: +82-2-3399-1617, E-mail: cheongjh@syu.ac.kr

Yeni Kim, MD, PhD

Department of Child and Adolescent Psychiatry, National Center of Mental Health, 127 Yongmasan-ro, Gwangjin-gu, Seoul 04933, Republic of Korea

Tel:+82-2-2204-0138, Fax: +82-2-2204-0355, E-mail: yenikim@korea.kr

*These authors contributed equally to this work.

(c) This is an Open Access article distributed under the terms of the Creative Commons Attribution Non-Commercial License (https://creativecommons.org/licenses/by-nc/4.0) which permits unrestricted non-commercial use, distribution, and reproduction in any medium, provided the original work is properly cited. 
peractivity. ${ }^{7}$ SHR showed differences in molecules regulating synaptic plasticity and in axon and dendrite development compared to WKY. ${ }^{8}$ Decreased expression in synaptotagmin, Munc18, synaptosomal-associated protein, Brain-derived neurotrophic factor and increased expression in the dopamine transporter, syntaxin and synaptophysin were reported in SHR compared to WKY. ${ }^{9}$

Psychostimulants such as methylphenidate (MPH), are the mainstay of treatment for ADHD. Previous reports show a delay in regional cortical maturation in $\mathrm{ADHD}$, most prominently in prefrontal regions important for the control of attention and motor planning. ${ }^{10}$ Neuroanatomical analysis has also revealed delayed maturation in the cortical regions of ADHD patients. ${ }^{10}$ Moreover, the prefrontal cortex is posited to play a prominent role in the therapeutic actions of lowdose stimulants. ${ }^{11}$ Another study showed increased glucose uptake in the cerebral cortex, basal hyperactivity, and paradoxical decreased locomotion after chronic methylphenidate injection. ${ }^{12}$ Therefore, we hypothesized that MPH may mediate therapeutic effects by restoring the molecular differences between WKY in the prefrontal cortex of SHR.

Cyclin dependent kinase 5 (Cdk5) is a serine/threonine kinase, which requires association with p35 for activation. ${ }^{13}$ The ratio of p25/p35 is also important in the regulation of Cdk5. ${ }^{14}$ The BGEE gene expression (https://bgee.org) database $^{15}$ reports that Cdk5 is expressed in the frontal cortex and dorsolateral prefrontal cortex of human brain, brain areas which showed early maturation in ADHD children compared to children who develop normally. ${ }^{10,16}$ In comparison, Cdk5 was not detected in the primary motor cortex. ${ }^{15}$ In one study, p35 deficient mice which have non-functional Cdk5 as the consequence of p35 deficiency, were reported to exhibit hyperactivity, ${ }^{17}$ a core feature of ADHD. Dysfunction of Cdk5 during development may affect the dopamine pathway, a pathway also strongly implicated in ADHD etiology. ${ }^{12}$ It has also been suggested that Cdk5 mediates these actions through auxiliary control of synaptic vesicle release and upstream synthesis of dopamine in the forebrain. ${ }^{18}$

Studies have linked C $\mathrm{dk} 5$ to Tropomyosin receptor kinase (TrkB) and Brain-derived neurotrophic factor-stimulated dendrite growth, ${ }^{19}$ moesin-controlled neuronal spine formation, ${ }^{20}$ and collapsin response mediator protein-2 (CRMP-2) regulated specification of neuronal axon/dendrite fate. ${ }^{21,22} \mathrm{Cdk} 5$ also forms a complex with Munc18 (otherwise known as syntaxin binding protein $)^{23}$ to regulate soluble N-ethylmaleimidesensitive factor attachment protein receptor (SNARE) complex, which includes syntaxin 1A (STX1A) and synaptosomalassociated protein 25 (SNAP25). ${ }^{24}$

In this study, we hypothesized that MPH can eliminate the molecular differences in WKY and SHR. Cdk5 and molecu- lar substrates of Cdk5, which play a pivotal role in synaptic plasticity and in axon and dendrite, were examined in the modulation of therapeutic effects of MPH in SHRs (a model for $\mathrm{ADHD}$ ) (Figure 1A).

\section{METHODS}

\section{Animal treatment}

Protocols for animal use were reviewed and approved by the Institutional Animal Care and Use Committee at the Sahmyook University (No. SYUIACUC 2015-005, SYUIACUC 2016-002) and were in accordance with National Institute of Health guidelines. Male 4 weeks old, SHR and WKY (Charles River, Japan) were used. Vehicle-treated animals were injected intraperitoneally with distilled water for 7 days, and $\mathrm{MPH}$-treated animals were injected intraperitoneally with MPH (2 mg/kg, i.p.) for 7 consecutive days during the adolescent period (post-natal days 29-35). The dosage was decided based on a previous study which showed that $2.5 \mathrm{mg} /$ $\mathrm{kg} \mathrm{MPH}$ improves attentional set-shifting at in SHRs. ${ }^{25} \mathrm{We}$ compared between vehicle-treated SHRs, vehicle-treated WKYs and MPH-treated SHRs. The animals were given access to food and water ad libitum and housed in a 12 hour light/dark cycle. The controls were given same volume of vehicle (distilled water) injections intraperitoneally as the MPH treated groups.

\section{Western blotting analysis}

Animals were sacrificed at $24 \mathrm{~h}$ after last injection of $\mathrm{MPH}$, and the rat brain was dissected on ice. The prefrontal cortex, striatum and hippocampus tissue were removed and homogenized in lysate buffer; $50 \mathrm{mM}$ Tris-base ( $\mathrm{pH}$ 7.5), $150 \mathrm{mM}$ $\mathrm{NaCl}, 2 \mathrm{mM}$ EDTA, 1\% glycerol, $10 \mathrm{mM}$ NaF, $10 \mathrm{mM} \mathrm{Na}$ pyrophosphate, $1 \%$ NP-40, and protease inhibitors cocktail. Protein concentration was determined by Bradford (Bio-Rad, Hercules, CA, USA) method. Thirty micrograms of cell lysates were electrophoresed in SDS-polyacrylamide gels (SDSPAGE) and transferred to nitrocellulose membranes, which were then incubated with anti-SNAP25 (1:1,000) (Santa Cruz Biotechnology, Dallas, TX, USA), anti-TrkB (1:1,000), antiCdk5 (1:1,000), anti-CRMP2 (1:1,000), anti-STX1A (1:1,000), anti- $\beta$-actin (1:1,000), anti-p35/p25 (1:1,000), anti-Munc18 $(1: 1,000)$, and anti-Moesin $(1: 2,000)^{26}$ antibodies for $16 \mathrm{~h}$ at $4^{\circ} \mathrm{C}$. After washing with TBS-T $(0.05 \%)$, the blots were incubated with horseradish peroxidase-conjugated anti-rabbit $(1: 3,000)$ or anti-mouse IgG (1:3,000) (Pierce Biotechnology, Rockford, IL, USA), and the bands were visualized using the ECL system (Pierce Biotechnology, Rockford). Band images were obtained by using a Molecular Imager ChemiDoc XRS+ (Bio-Rad, Hercules) and band intensity was analyzed using Image Lab ${ }^{\mathrm{TM}}$ software version 2.0.1 (Bio-Rad, Hercules). 


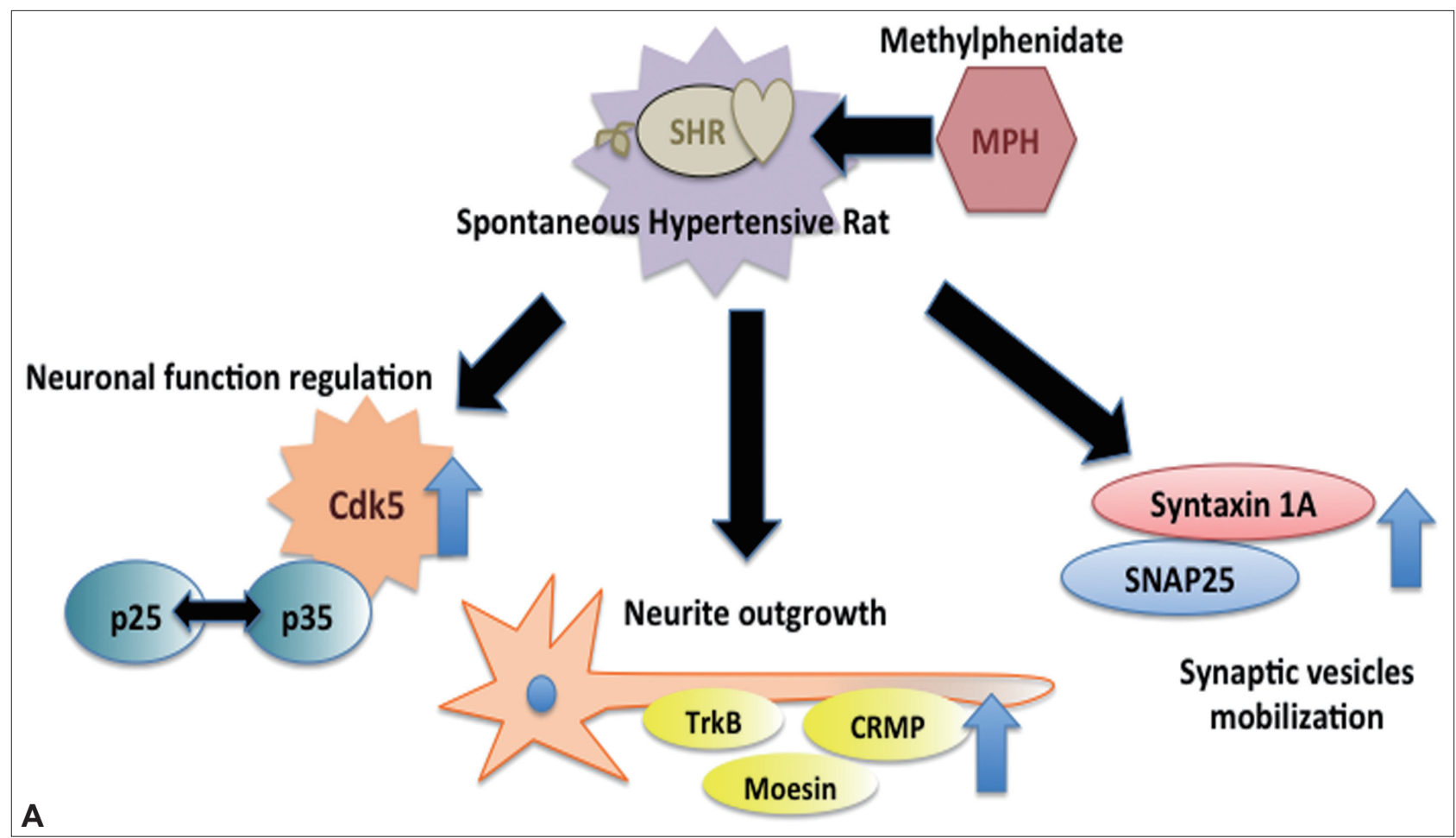

Figure 1. MPH replenishes the decreased Cdk5, TrkB, STX1A, and SNAP25 and decreases the p25/p35 ratio in prefrontal cortex of SHRs. Schematic presentation of molecules involved in effect of MPH in prefrontal cortex of SHRs (A), each brain lysates were immunoblotted with Cdk5 (B), p25 (C), p35 (C), TrkB (D), Moesin (E), CRMP-2 (F) STX1A (G), SNAP25 (H), and Munc18 (I) antibodies. The intensity of each band was normalized to that of $\beta$-actin and presented in bar graphs. The values represent the means $\pm S D(n=3-5)$. ${ }^{*} p<0.05,{ }^{* *} p<0.01$.

\section{Statistical analysis}

All statistical analyses were conducted with SPSS (version 19, Somers, NY, USA). The intensity of each band was normalized to that of $\beta$-actin (data not shown) and shown as mean \pm standard deviation (error bar). Group comparison was made using analysis of variance test (ANOVA) and post-hoc comparison was done using LSD method. The p-value of 0.05 or less was considered statistically significant.

\section{RESULTS}

\section{Cdk5 proteins and $\mathrm{p} 25 / \mathrm{p} 35$ ratio}

Cdk5 levels (Figure 1B) in the prefrontal cortex of vehicletreated SHR (69.9 $\pm 9.1 \%)$ were significantly decreased compared to Cdk5 levels in vehicle-treated WKY $(127.7 \pm 26.3 \%$, $\mathrm{p}<0.01)$. After 7 days of treatment with $2 \mathrm{mg} / \mathrm{kg} \mathrm{MPH,} \mathrm{Cdk5}$

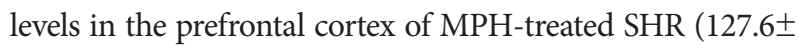
$10.4 \%)$ were increased compared to $\mathrm{Cdk} 5$ levels in vehicletreated SHR $(69.9 \pm 9.1 \%, \mathrm{p}<0.01)$ and to the levels expressed in WKYs. In the hippocampus, Cdk5 levels in vehicle-treated SHR $(82.1 \pm 13.1 \%)$ were increased compared to Cdk5 levels in vehicle-treated WKY $(112.4 \pm 16.9 \%, \mathrm{p}<0.05)$ and decreased

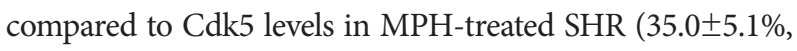
$\mathrm{p}<0.01)$. In the prefrontal cortex, the ratio of $\mathrm{p} 25 / \mathrm{p} 35$ was sig- nificantly decreased in vehicle-treated SHR $(0.63 \pm 0.18 \%)$ compared to vehicle-treated WKY $(1.01 \pm 0.05 \%, \mathrm{p}<0.01)$, and p25/p35 was significantly decreased in MPH-treated SHR $(0.20 \pm 0.07 \%)$ compared to vehicle-treated SHR $(0.63 \pm 0.18 \%$, $\mathrm{p}<0.01$ ) (Figure 1C). We observed that in striatum, p25/p35 was significantly decreased in vehicle-treated SHR $(0.45 \pm$ $0.22 \%)$ compared to vehicle-treated WKY $(1.38 \pm 0.10 \%, \mathrm{p}<$ 0.01 ), but there was no significant difference in $\mathrm{p} 25 / \mathrm{p} 35$ in MPH-treated SHR $(0.19 \pm 0.03 \%)$ compared to vehicle-treated SHR $(0.45 \pm 0.22 \%)$. In the hippocampus, there was no difference in the ratio of p25/p35 between groups (vehicletreated WKY $1.25 \pm 0.13 \%$, vehicle-treated SHR $1.18 \pm 0.73 \%$, MPH-treated SHR 0.25 $\pm 0.12 \%$ ).

\section{TrkB, Moesin and CRMP-2 proteins}

In the prefrontal cortex, TrkB levels (Figure 1D) in vehicletreated SHRs $(67.5 \pm 25.6 \%)$ were significantly decreased compared to TrkB levels in vehicle-treated WKY (107.4 $\pm 12.9 \%$, $\mathrm{p}<0.05)$. After MPH treatment, the TrkB levels (MPH-treated SHR 105.2 $\pm 7.2 \%$ ) in the prefrontal cortex were increased compared to TrkB levels in vehicle-treated SHR $(67.5 \pm 25.6 \%$, $\mathrm{p}<0.05)$. TrkB (MPH-treated SHR 318.4 $\pm 106.3 \%$ compared to vehicle-treated SHR $86.3 \pm 39.0 \%, \mathrm{p}<0.01)$ and Moesin (Figure 1E) (MPH-treated SHR 152.4 $\pm 42.8 \%$ compared to 


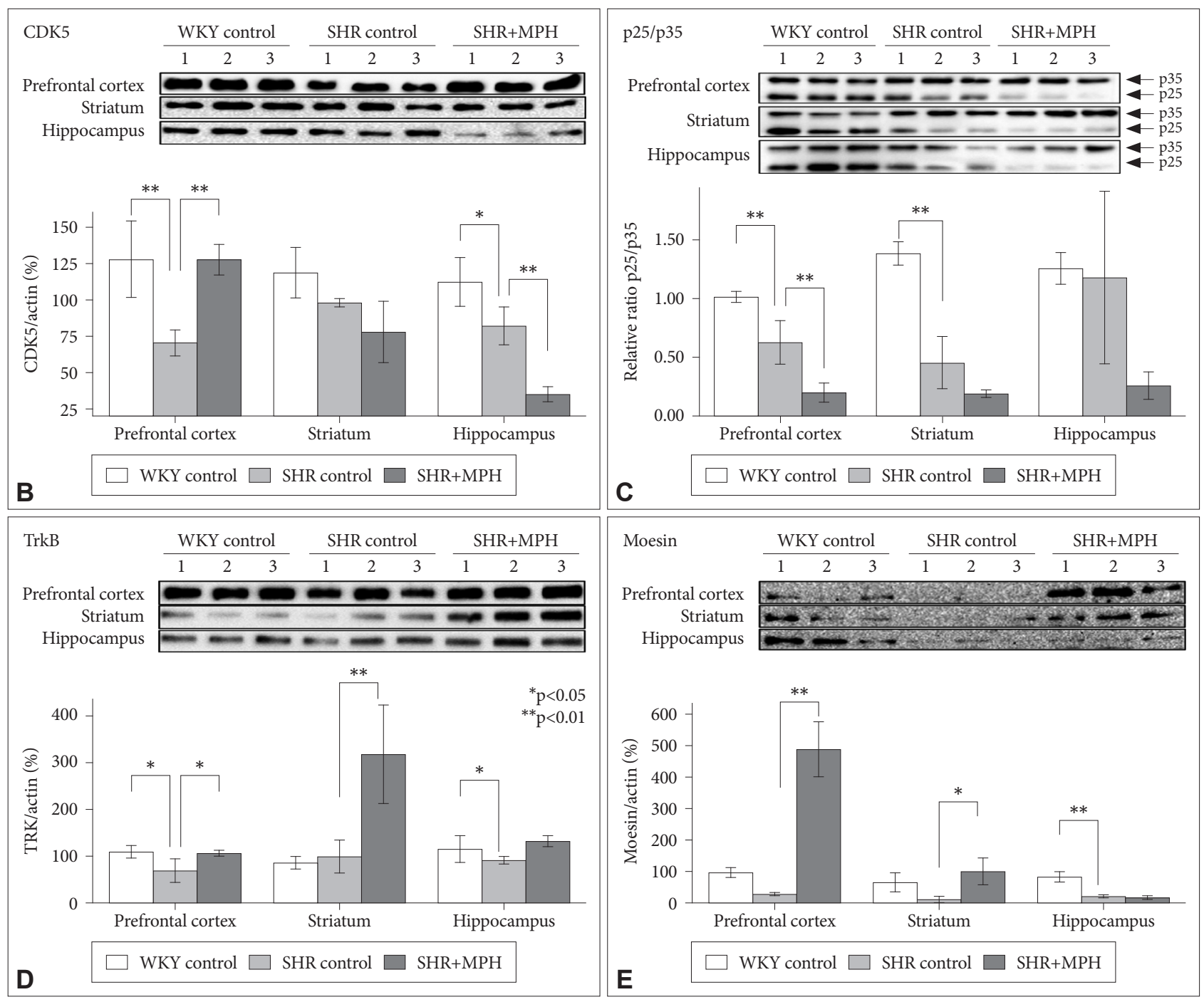

Figure 1. MPH replenishes the decreased Cdk5, TrkB, STX1A, and SNAP25 and decreases the p25/p35 ratio in prefrontal cortex of SHRs. Schematic presentation of molecules involved in effect of MPH in prefrontal cortex of SHRs $(A)$, each brain lysates were immunoblotted with Cdk5 (B), p25 (C), p35 (C), TrkB (D), Moesin (E), CRMP-2 (F) STX1A (G), SNAP25 (H), and Munc18 (I) antibodies. The intensity of each band was normalized to that of $\beta$-actin and presented in bar graphs. The values represent the means \pm SD $(n=3-5)$. ${ }^{*} p<0.05,{ }^{* *} p<0.01$.

vehicle-treated SHR 17.6 $\pm 9.1 \%, \mathrm{p}<0.05)$ levels in SHRs were significantly increased in the striatum after MPH treatment. We observed that TrkB (vehicle-treated SHR $82.1 \pm 13.1 \%$ compared to vehicle-treated WKY $112.4 \pm 16.9 \%, \mathrm{p}<0.05)$, and Moesin (vehicle-treated SHR $27.8 \pm 4.1 \%$ compared to vehicle-treated WKY $100.0 \pm 17.0 \%, \mathrm{p}<0.01$ ) levels were also decreased in the hippocampus in vehicle-treated SHRs compared to vehicle-treated WKYs, and the MPH treatment did not increase the expression levels of these proteins in $\mathrm{MPH}-$ treated SHRs compared to vehicle-treated SHRs. CRMP-2 (Figure $1 \mathrm{~F}$ ) was also significantly decreased in the prefrontal cortex of vehicle-treated SHRs $(61.1 \pm 2.6 \%)$ compared to vehicle-treated WKYs $(98.0 \pm 15.4 \%, \mathrm{p}<0.01)$. However, the increase in CRMP-2 after MPH treatment was statistically insignificant.

\section{STX1A, SNAP25, and Munc18 proteins}

STX1A (Figure 1G) (vehicle-treated SHR 55.3 $\pm 7.0 \%$ compared to $101.0 \pm 6.0 \%$ of vehicle-treated $\mathrm{WKY}, \mathrm{p}<0.05$ ), SNAP25 (Figure $1 \mathrm{H}$ ) (vehicle-treated SHR $27.5 \pm 8.1 \%$ compared to $106.8 \pm 24.8 \%$ of vehicle-treated WKY, $\mathrm{p}<0.05$ ), and Munc18 (Figure 1I), (vehicle-treated SHR $83.4 \pm 8.1 \%$ compared to $110.4 \pm 11.2 \%$ of vehicle-treated WKY, $\mathrm{p}<0.05$ ) levels were significantly decreased in the prefrontal cortex of vehicletreated SHR compared to the levels of these proteins in vehicle-treated WKY. After MPH treatment, the STX1A (MPHtreated SHR $76.5 \pm 15.0 \%$ compared to $55.3 \pm 7.0 \%$ of vehicletreated SHRs, $\mathrm{p}<0.01)$ and SNAP25 (MPH-treated SHR $212.1 \pm 54.2 \%$ compared to $27.5 \pm 8.1 \%$ of vehicle-treated SHRs, $\mathrm{p}<0.01)$ levels in the prefrontal cortex of MPH-treated SHR were increased compared to the levels of these proteins 


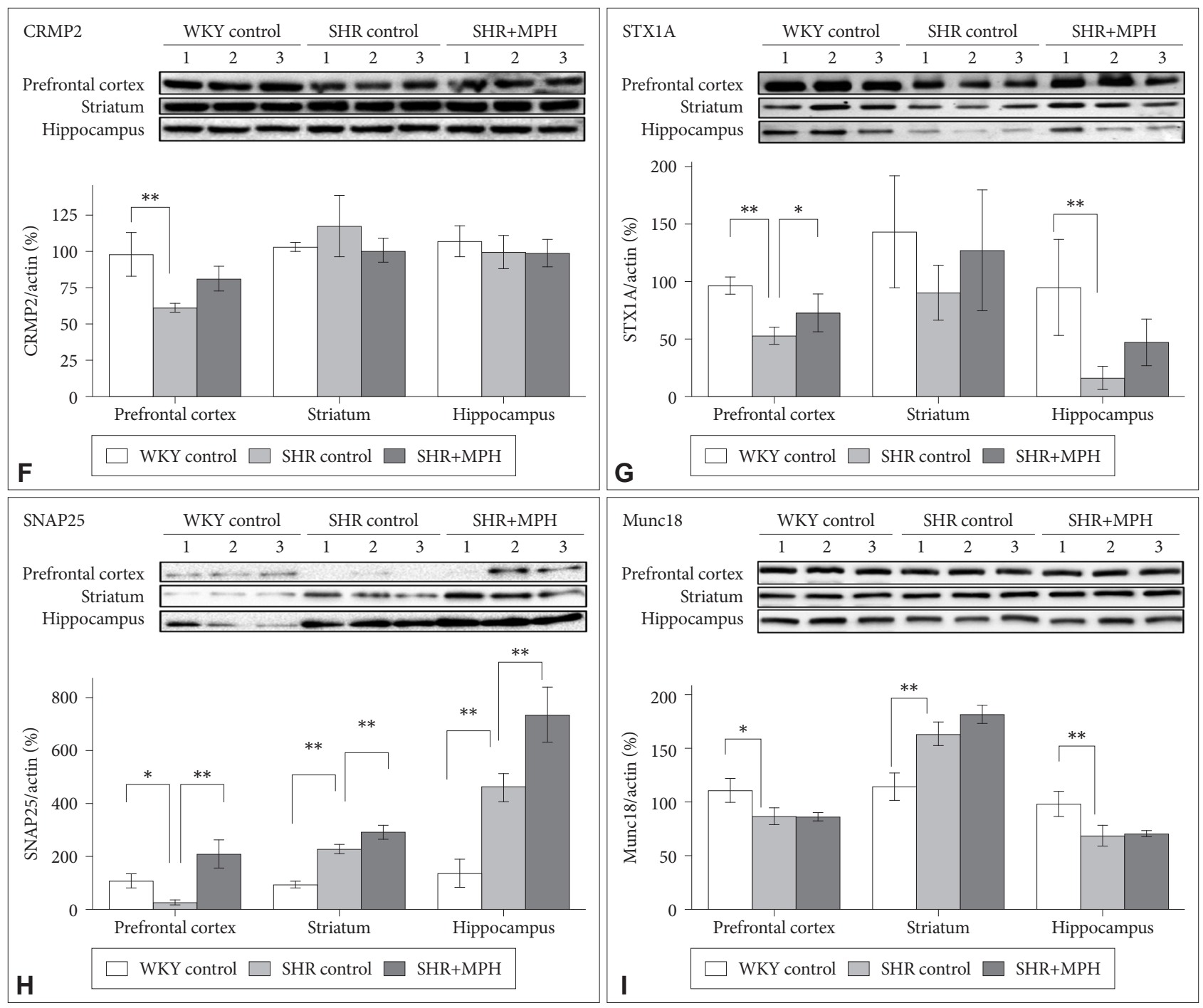

Figure 1. MPH replenishes the decreased Cdk5, TrkB, STX1A, and SNAP25 and decreases the p25/p35 ratio in prefrontal cortex of SHRs. Schematic presentation of molecules involved in effect of MPH in prefrontal cortex of SHRs (A), each brain lysates were immunoblotted with Cdk5 (B), p25 (C), p35 (C), TrkB (D), Moesin (E), CRMP-2 (F) STX1A (G), SNAP25 (H), and Munc18 (I) antibodies. The intensity of each band was normalized to that of $\beta$-actin and presented in bar graphs. The values represent the means $\pm S D(n=3-5)$. ${ }^{*} p<0.05$, ${ }^{* *} p<0.01$.

in WKYs. SNAP25 levels were significantly increased in the striatum (vehicle-treated SHR $227.2 \pm 20.0 \%$ compared to $93.5 \pm 13.0 \%$ of vehicle-treated WKY, $\mathrm{p}<0.01)$ and the hippocampus (vehicle-treated SHR $461.7 \pm 50.0 \%$ compared to $137.0 \pm 54.8 \%$ of vehicle-treated WKY, $<<0.01$ ) in vehicletreated SHR compared to SNAP25 levels in vehicle-treated WKY. MPH treatment significantly increased the expression levels of SNAP25 in the striatum (MPH-treated SHR 293.4 $\pm 25.8 \%$ compared to $227.2 \pm 20.0 \%$ of vehicle-treated SHRs, $\mathrm{p}<0.01$ ) and the hippocampus (MPH-treated SHR 732.0 \pm $102.2 \%$ compared to $461.7 \pm 50.0 \%$ of vehicle-treated SHRs, $\mathrm{p}<0.01$ ). STX1A (vehicle-treated SHR $17.9 \pm 8.8 \%$ compared to $100.2 \pm 38.4 \%$ of vehicle-treated $\mathrm{WKY}, \mathrm{p}<0.05$ ), and Munc18 (vehicle-treated SHR $68.7 \pm 9.8 \%$ compared to 90.6 $\pm 9.3 \%$ of vehicle-treated WKY, $\mathrm{p}<0.05)$ were also decreased in the hippocampus of vehicle-treated SHRs compared to vehicle-treated WKY, and MPH treatment did not increase the expression levels of these proteins in MPH-treated SHR compared to those of vehicle-treated SHRs.

\section{DISCUSSION}

We investigated the role of $\mathrm{Cdk} 5$ and the substrates of Cdk5 in mediating the therapeutic effects of MPH. We observed that decreased expression of Cdk5, TrkB, STX1A, and SNAP25, were restored to the levels in WKYs after MPH treatment in the prefrontal cortex of SHRs. These results suggest that MPH restores Cdk5 expression in SHRs to the expression level in WKYs.

In this study, the relative p25/p35 ratio was decreased after 
$\mathrm{MPH}$ treatment due to a decrease in p25, rather than an increase in $\mathrm{p} 35$. The result suggests that $\mathrm{MPH}$ may regulate Cdk5 by decreasing the relative $\mathrm{p} 25 / \mathrm{p} 35$ ratio. Previous studies have shown that Cdk5/p25 association causes aberrant hyperphosphorylation of substrates of Cdk $5,{ }^{27}$ and that reducing Cdk5 activity through knockout of p35 induces behavioral hyperactivity in mice. ${ }^{12}$

In a post-mortem study, genetic variation of SNAP25 confers risk for ADHD and reduces the expression of the SNAP25 transcript in a region of the brain that is critical for the regulation of attention and inhibition. ${ }^{28}$ Coloboma mice, which are mice with a 50\% reduction in SNAP25 protein levels, manifest locomotor hyperactivity. ${ }^{29,30}$ The hyperactivity manifested by Coloboma mice is ameliorated by low-dose amphetamine but not by MPH. ${ }^{29,30}$ In our study similar results were observed. MPH increased SNAP25 protein levels in SHRs to WKY levels, which is not possible for mice with deletions in the SNAP25 gene. The paradoxical response to MPH in Coloboma mice, along with our observations, suggest that $\mathrm{MPH}$ may exert its therapeutic effect through an increase in the level of SNAP25 protein in the prefrontal cortex.

$\operatorname{TrkB}$ is a protein with major function in neurite growth, which is also regulated by $\mathrm{Cdk} 5$. TrkB-signaling gene knockout $^{31}$ showed impaired hippocampal-dependent learning deficits in mice, similar to the learning impairment reported in ADHD. STX1A directly interacts with dopamine and regulates dopamine-mediated amphetamine-induced efflux. ${ }^{32}$ Therefore, altered STX1A function may modulate dopamine systems, contributing to ADHD symptoms. ${ }^{33}$ In this study, the expression of TrkB was decreased in vehicle-treated SHRs compared to vehicle-treated WKYs. Moreover, STX1A which was decreased in vehicle-treated SHRs compared to vehicletreated WKYs, were also restored to the levels in WKYs after MPH treatment. These results suggest that synaptic vesicle mobilization and neurite outgrowth pathways may play an important role in the therapeutic effects of $\mathrm{MPH}$.

The results of this study, showed that Cdk5, TrkB, STX1A, and SNAP25 were involved in the modulation of MPH effects in the prefrontal cortex of SHRs, suggesting that synaptic vesicle mobilization and neurite outgrowth pathways may play an important role in ADHD pathophysiology and treatment.

\section{Acknowledgments}

This study was supported by grant from the Korean Health Technology R\&D Project, Ministry of Health \& Welfare (A120013) and intramural grant from the National Center for Mental Health (No. 2017-08), Republic of Korea.

\section{Conflicts of Interest}

The authors have no potential conflicts of interest to disclose.

\section{Author Contributions}

Conceptualization: Yeni Kim, Songhee Jeon, Jae Hoon Cheong. Data curation: Yeni Kim, Songhee Jeon, Ha Jin Jeong, Ike dela Peña, Hee Jin Kim. Formal analysis: Yeni Kim, Songhee Jeon, Ike dela Peña. Funding acquisition: Yeni Kim, Bung-Nyun Kim, Doug Hyun Han, Jae Hoon Cheong. Investigation: Yeni Kim, Songhee Jeon, Jae Hoon Cheong, Ike dela Peña. Methodology: Yeni Kim, Songhee Jeon, Jae Hoon Cheong, Ike dela Peña. Project administration: Hee Jin Kim, Seong Mi Lee. Resources: Hee Jin Kim, Seong Mi Lee, Ha Jin Jeong. Software: Hee Jin Kim, Seong Mi Lee. Supervision: Yeni Kim, Bung-Nyun Kim, Doug Hyun Han, Jae Hoon Cheong. Validation: Yeni Kim, Songhee Jeon, Ha Jin Jeong, Seong Mi Lee. Visualization: Ha Jin Jeong, Seong Mi Lee. Writing_original draft: Yeni Kim, Songhee Jeon, Seong Mi Lee. Writing_review \& editing: Yeni Kim, Songhee Jeon, Bung-Nyun Kim, Doug Hyun Han, Jae Hoon Cheong.

\section{ORCID iDs}

\author{
Jae Hoon Cheong https://orcid.org/0000-0001-5654-5868 \\ Yeni Kim \\ Songhee Jeon \\ https://orcid.org/0000-0002-2624-8026 \\ https://orcid.org/0000-0003-4978-3416
}

\section{REFERENCES}

1. Limbers CA, Ripperger-Suhler J, Boutton K, Ransom D, Varni JW. A comparative analysis of health-related quality of life and family impact between children with ADHD treated in a general pediatric clinic and a psychiatric clinic utilizing the PedsQL. J Atten Disord 2011;15:392-402.

2. Froehlich TE, Lanphear BP, Epstein JN, Barbaresi WJ, Katusic SK, Kahn RS. Prevalence, recognition, and treatment of attention-deficit/ hyperactivity disorder in a national sample of US children. Arch Pediatr Adolesc Med 2007;161:857-864.

3. Okamoto K, Aoki K. Development of a strain of spontaneously hypertensive rats. Jpn Circ J 1963;27:282-293.

4. Miller EM, Pomerleau F, Huettl P, Russell VA, Gerhardt GA, Glaser PE. The spontaneously hypertensive and Wistar Kyoto rat models of ADHD exhibit sub-regional differences in dopamine release and uptake in the striatum and nucleus accumbens. Neuropharmacology 2012;63:1327-1334.

5. Russell VA. Overview of animal models of attention deficit hyperactivity disorder (ADHD). Curr Protoc Neurosci 2011; Chapter 9: Unit9. 35.

6. Sagvolden T, Johansen EB. Rat models of ADHD. Curr Top Behav Neurosci 2012;9:301-315.

7. Sagvolden T. Behavioral validation of the spontaneously hypertensive rat (SHR) as an animal model of attention-deficit/hyperactivity disorder (AD/HD). Neurosci Biobehav Rev 2000;24:31-39.

8. Sagvolden T, Johansen EB, Woien G, Walaas SI, Storm-Mathisen J, Bergersen LH, et al. The spontaneously hypertensive rat model of ADHD--the importance of selecting the appropriate reference strain. Neuropharmacology 2009;57:619-626.

9. DasBanerjee T, Middleton FA, Berger DF, Lombardo JP, Sagvolden T, Faraone SV. A comparison of molecular alterations in environmental and genetic rat models of ADHD: a pilot study. Am J Med Genet B Neuropsychiatr Genet 2008;147B:1554-1563.

10. Shaw P, Eckstrand K, Sharp W, Blumenthal J, Lerch JP, Greenstein D, et al. Attention-deficit/hyperactivity disorder is characterized by a delay in cortical maturation. Proc Natl Acad Sci U S A 2007; 104: 1964919654.

11. Castellanos FX, Tannock R. Neuroscience of attention-deficit/hyperactivity disorder: the search for endophenotypes. Nat Rev Neurosci 2002;3:617-628.

12. Drerup JM, Hayashi K, Cui H, Mettlach GL, Long MA, Marvin M, et al. Attention-deficit/hyperactivity phenotype in mice lacking the cyclin-dependent kinase 5 cofactor p35. Biol Psychiatry 2010;68:11631171.

13. Kawauchi T. Cellullar insights into cerebral cortical development: fo- 
cusing on the locomotion mode of neuronal migration. Front Cell Neurosci 2015;9:394.

14. Huang T, Fang L, Lin Z, Huang E, Ye Q. Increase of p25 associated with cortical neuronal death induced by hypoxia. Biochem Biophys Res Commun 2016;477:932-936.

15. BGEE: Gene Expression data in animals. Available at: https://bgee.org. Accessed June 1, 2019.

16. Maitra S, Chatterjee M, Sinha S, Mukhopadhyay K. Cyclin-dependent Kinase 5: Novel role of gene variants identified in ADHD. Sci Rep 2017;7:6828.

17. Krapacher FA, Mlewski EC, Ferreras S, Pisano V, Paolorossi M, Hansen $\mathrm{C}$, et al. Mice lacking p35 display hyperactivity and paradoxical response to psychostimulants. J Neurochem 2010;114:203-214.

18. Chergui K, Svenningsson P, Greengard P. Cyclin-dependent kinase 5 regulates dopaminergic and glutamatergic transmission in the striatum. Proc Natl Acad Sci U S A 2004;101:2191-2196.

19. Cheung ZH, Chin WH, Chen Y, Ng YP, Ip NY. Cdk5 is involved in BDNF-stimulated dendritic growth in hippocampal neurons. PLoS Biol 2007;5:e63.

20. Sanchez AM, Flamini MI, Fu XD, Mannella P, Giretti MS, Goglia L, et al. Rapid signaling of estrogen to WAVE1 and moesin controls neuronal spine formation via the actin cytoskeleton. Mol Endocrinol 2009; 23:1193-1202.

21. Brown M, Jacobs T, Eickholt B, Ferrari G, Teo M, Monfries C, et al. Alpha2-chimaerin, cyclin-dependent Kinase 5/p35, and its target collapsin response mediator protein- 2 are essential components in semaphorin 3A-induced growth-cone collapse. J Neurosci 2004;24:89949004.

22. Jin X, Sasamoto K, Nagai J, Yamazaki Y, Saito K, Goshima Y, et al. Phosphorylation of CRMP2 by Cdk5 Regulates Dendritic Spine Development of Cortical Neuron in the Mouse Hippocampus. Neural Plast 2016;2016:6790743.

23. Fletcher AI, Shuang R, Giovannucci DR, Zhang L, Bittner MA, Stuen- kel EL. Regulation of exocytosis by cyclin-dependent kinase 5 via phosphorylation of Munc18. J Biol Chem 1999;274:4027-4035.

24. Sutton RB, Fasshauer D, Jahn R, Brunger AT. Crystal structure of a SNARE complex involved in synaptic exocytosis at $2.4 \mathrm{~A}$ resolution. Nature 1998;395:347-353.

25. Cao AH, Yu L, Wang YW, Wang JM, Yang LJ, Lei GF. Effects of methylphenidate on attentional set-shifting in a genetic model of attentiondeficit/hyperactivity disorder. Behav Brain Funct 2012;8:10.

26. Jeon S, Kim S, Park JB, Suh PG, Kim YS, Bae CD, et al. RhoA and Rho kinase-dependent phosphorylation of moesin at Thr-558 in hippocampal neuronal cells by glutamate. J Biol Chem 2002;277:1657616584.

27. Lee MS, Kwon YT, Li M, Peng J, Friedlander RM, Tsai LH. Neurotoxicity induces cleavage of p35 to p25 by calpain. Nature 2000;405:360-364.

28. Hawi Z, Matthews N, Wagner J, Wallace RH, Butler TJ, Vance A, et al. DNA variation in the SNAP25 gene confers risk to ADHD and is associated with reduced expression in prefrontal cortex. PLoS One 2013; 8:e60274.

29. Hess EJ, Jinnah HA, Kozak CA, Wilson MC. Spontaneous locomotor hyperactivity in a mouse mutant with a deletion including the Snap gene on chromosome 2. J Neurosci 1992;12:2865-2874.

30. Hess EJ, Collins KA, Wilson MC. Mouse model of hyperkinesis implicates SNAP-25 in behavioral regulation. J Neurosci 1996;16:3104-3111.

31. Minichiello L, Korte M, Wolfer D, Kuhn R, Unsicker K, Cestari V, et al. Essential role for TrkB receptors in hippocampus-mediated learning. Neuron 1999;24:401-414.

32. Binda F, Dipace C, Bowton E, Robertson SD, Lute BJ, Fog JU, et al. Syntaxin $1 \mathrm{~A}$ interaction with the dopamine transporter promotes amphetamine-induced dopamine efflux. Mol Pharmacol 2008;74:11011108 .

33. Faraone SV, Khan SA. Candidate gene studies of attention-deficit/hyperactivity disorder. J Clin Psychiatry 2006;67(Suppl 8):13-20. 